\title{
ON PAIRS OF ADDITIVE CUBIC EQUATIONS
}

\author{
By R. C. VAUGHAN
}

[Received 5 August 1975]

\section{Introduction}

Davenport and Lewis [3] have shown that if $c_{1}, \ldots, c_{n}, d_{1}, \ldots, d_{n}$ are rational integers, then the simultaneous equations

$$
\left.\begin{array}{l}
F=c_{1} x_{1}{ }^{3}+\ldots+c_{n} x_{n}^{3}=0, \\
G=d_{1} x_{1}^{3}+\ldots+d_{n} x_{n}^{3}=0
\end{array}\right\}
$$

have a non-trivial solution (that is, a rational integral solution with not all the $x_{i}$ zero) provided that $n \geqslant 18$. Cook [1] has shown that the 18 can be replaced by 17 .

The purpose of this paper is to establish the following theorem.

Theorem. The two simultaneous equations (1.1) have a non-trivial solution whenever $n \geqslant 16$.

An example of Davenport and Lewis [3, (4) and (5)] with $n=15$ and having no non-trivial solution implies that the above theorem is essentially best possible.

As with previous work on this subject, we use a modified form of the Hardy-Littlewood method. The principal difficulty lies as usual with the minor arcs. The new idea is to give a major arc treatment to those parts of the minor arcs which give the greatest difficulty in the DavenportLewis argument. However, in order to succeed with this approach it is also necessary to make use of a generalization of Cook's observation concerning Hua's lemma. There is further a rather delicate question as to the number of times that the same ratio occurs among the ratios $c_{i} / d_{i}$ and the manner in which they are distributed.

\section{The rearrangement of the variables}

If for some $j$ both $c_{j}$ and $d_{j}$ are zero, then the theorem is trivial. Therefore we can assume that for each $j$ one of $c_{j}$ and $d_{j}$ is non-zero. Thus the ratio $c_{j} / d_{j}$ always exists (in the extended real number system) and we say that two ratios $c_{i} / d_{i}$ and $c_{j} / d_{j}$ are equal if $c_{i} d_{j}=c_{j} d_{i}$. For brevity, let $r_{i}$ denote $c_{i} / d_{i}$. To prove the theorem it clearly suffices to consider only the case where $n=16$. An argument of Davenport and Lewis [3, p. 115] (the 18 there can be replaced trivially by 16) disposes at once of 
the case in which seven or more of the ratios $r_{i}$ are equal. Thus we can assume that no ratio $r_{i}$ is repeated more than six times.

The following lemma is the basis for an appropriate rearrangement of the variables.

LEMMA 1. Suppose that among the ratios $r_{1}, \ldots, r_{16}$ no ratio is repeated more than six times. Then the suffices $1, \ldots, 16$ can be rearranged into two disjoint sets $\mathscr{A}, \mathscr{B}$ with ten and six elements respectively and such that

(i) among the ratios $r_{j}$ with $j \in \mathscr{A}$ no ratio is repeated more than four times,

(ii) the ratios $r_{j}$ with $j \in \mathscr{B}$ take on at least three distinct values and no value occurs more than twice.

Proof. Suppose that exactly $m$ distinct ratios occur among $r_{1}, \ldots, r_{16}$. Group the same ratios together so that there are $m$ groups, the $j$ th group has $l_{j}$ members and

$$
6 \geqslant l_{1} \geqslant l_{2} \geqslant \ldots \geqslant l_{m} \geqslant 1 .
$$

Clearly $m \geqslant 3, l_{3} \leqslant 5$, and if $m \geqslant 4$, then $l_{4} \leqslant 4$. Let $\mathscr{D}_{j}$ denote the suffices corresponding to the $j$ th group. If $l_{2}=1$, then place $\min \left(4, l_{1}\right)$ members of $\mathscr{D}_{1}$ in $\mathscr{A}$ and the rest (if any) in $\mathscr{B}$. Then distribute the members of $\mathscr{D}_{2}, \ldots, \mathscr{D}_{m}$ at random.

If $l_{2} \geqslant 2$, then form $\mathscr{B}$ by taking two elements from $\mathscr{D}_{1}$, two from $\mathscr{D}_{2}$, and two from $\mathscr{D}_{3}$ (unless $l_{3}=1$ in which case $m \geqslant 4$ and one takes two from $\left.\mathscr{D}_{3} \cup \mathscr{D}_{4}\right)$. The remaining suffices are placed in $\mathscr{A}$.

This completes the proof of the lemma.

We relabel the variables in the following way. The suffices in $\mathscr{A}$ are changed to $1, \ldots, 10$. Those in $\mathscr{B}$ are changed to $11, \ldots, 16$ in such a way that

or

$$
\left.\begin{array}{ll}
r_{11}=r_{13}, & r_{12}=r_{14}, \quad r_{11} \neq r_{12}, \quad r_{11} \neq r_{15}, \\
r_{11} \neq r_{16}, \quad r_{12} \neq r_{15}, \quad r_{12} \neq r_{16},
\end{array}\right\}
$$

or

$$
r_{11}, r_{12}, r_{13}, r_{14}, r_{15} \text { are distinct and } r_{15}=r_{16} \text {, }
$$

$$
r_{11}, \ldots, r_{16} \text { are distinct. }
$$

\section{Notation required in the proof}

Since the ratios $r_{1}, \ldots, r_{12}$ are not all equal we can choose non-zero real numbers $\eta_{1}, \ldots, \eta_{12}$ so that

$$
\left.\begin{array}{l}
c_{1} \eta_{1}+\ldots+c_{12} \eta_{12}=0 \\
d_{1} \eta_{1}+\ldots+d_{12} \eta_{12}=0
\end{array}\right\}
$$

Moreover, we can assume without loss of generality that $\eta_{i}>0$ $(i=1, \ldots, 12)$ since whenever necessary the $c_{i}, d_{i}$ can be replaced by 
$-c_{i},-d_{i}$, and $x_{i}^{3}$ by $\left(-x_{i}\right)^{3}$. Let

and

$$
\xi_{i}=\frac{1}{2} \eta_{i}^{1 / 3}, \quad \zeta_{i}=2 \eta_{i}^{1 / 3} \quad(i=1, \ldots, 12),
$$

$$
T_{i}(\gamma)=\sum_{\xi_{1} P<x<\zeta_{1} P} e\left(\gamma x^{3}\right),
$$

where $P$ is large (in terms of $\varepsilon, c_{1}, \ldots, c_{16}, d_{1}, \ldots, d_{16}, \eta_{1}, \ldots, \eta_{16}$ ) and $e(\alpha)=e^{2 \pi i \alpha}$.

We further write

$$
U(\gamma)=\sum_{P^{\mathrm{A} / 6}<x<2 P^{\mathrm{A} / 5}} e\left(\gamma x^{3}\right) .
$$

Our object is to estimate the number $\mathscr{N}(P)$ of solutions of (1.1) which satisfy

$$
\left.\begin{array}{l}
\xi_{i} P<x_{i}<\zeta_{i} P \quad(i=1, \ldots, 12), \\
P^{4 / 5}<x_{i}<2 P^{4 / 5} \quad(i=13, \ldots, 16) .
\end{array}\right\}
$$

Let $\alpha_{1}, \alpha_{2}$ be real variables,

and

$$
\begin{gathered}
\gamma_{i}=c_{i} \alpha_{1}+d_{i} \alpha_{2} \quad(i=1, \ldots, 16), \\
\delta=10^{-2},
\end{gathered}
$$

Then

$$
\eta=P^{-2-\delta} \text {. }
$$

$$
\mathscr{N}(P)=\int_{\eta}^{1+\eta} \int_{\eta}^{1+\eta} T_{1}\left(\gamma_{1}\right) \ldots T_{12}\left(\gamma_{12}\right) U\left(\gamma_{13}\right) \ldots U\left(\gamma_{16}\right) d \alpha_{1} d \alpha_{2} .
$$

The open square $(\eta, 1+\eta) \times(\eta, 1+\eta)$ is dissected in the following way. We denote a typical major arc by

where

$$
\mathfrak{M}\left(a_{1}, a_{2}, q\right)=\left\{\left(\alpha_{1}, \alpha_{2}\right):\left|q \alpha_{i}-a_{i}\right|<P^{-2-\delta}(i=1,2)\right\},
$$

$$
\left(a_{1}, a_{2}, q\right)=1 \text { and } 1 \leqslant a_{1}, a_{2} \leqslant q \leqslant P^{1-\delta} \text {. }
$$

The $\mathfrak{M}\left(a_{1}, a_{2}, q\right)$ are disjoint since, whenever $a / q \neq a^{\prime} / q^{\prime}$ and $q, q^{\prime} \leqslant P^{1-8}$, $\left|a / q-a^{\prime} / q^{\prime}\right| \geqslant 1 /\left(q q^{\prime}\right)>\left(1 / q+1 / q^{\prime}\right) P^{-2-\delta}$. Let $\mathfrak{M}$ denote the union of the major arcs, and $m$ the minor arcs,

$$
\mathfrak{m}=(\eta, 1+\eta) \times(\eta, 1+\eta) \backslash \mathfrak{M} .
$$

Throughout, $\varepsilon$ is a sufficiently small positive number and the implied constants in the $O, \ll$, and $\gg$ notations depend at most on $\varepsilon, c_{1}, \ldots, c_{16}$, $d_{1}, \ldots, d_{16}, \eta_{1}, \ldots, \eta_{16}$.

\section{The minor arcs}

\section{LEMMA 2. We have}

$$
\int_{0}^{1} \int_{0}^{1}\left|T_{11}\left(\gamma_{11}\right) T_{12}\left(\gamma_{12}\right) U\left(\gamma_{13}\right) \ldots U\left(\gamma_{16}\right)\right|^{2} d \alpha_{1} d \alpha_{2} \ll P^{26 / 5+\varepsilon} .
$$


Proof. This is essentially Lemma 19 of Davenport and Lewis [3]. There is a slight obscurity in the first line of their proof in that the reduction (in our notation) to the case where $r_{15}=r_{16}$ could conflict with the possibility that $r_{16}=r_{i}$ for $i=11,12,13$, or 14. However, in (2.1), (2.2), and (2.3) we have arranged that this conflict does not occur.

Lemma 3. Suppose that $1 \leqslant i, j \leqslant 10$ and $r_{i} \neq r_{j}$. Then

$$
\int_{0}^{1} \int_{0}^{1}\left|T_{i}\left(\gamma_{i}\right) T_{j}\left(\gamma_{j}\right)\right|^{8} d \alpha_{1} d \alpha_{2} \ll P^{10+8}
$$

This is Lemma 2 of Cook [1].

Let

$$
S(a, q)=\sum_{m=1}^{q} e\left(a m^{3} / q\right) .
$$

Lemma 4. Suppose that $(a, q)=1$. Then

$$
S(a, q) \ll q^{2 / 3} \text {. }
$$

This is Lemma 3 of Hardy and Littlewood [6].

Lemma 5. Suppose that $q \leqslant P^{1-8},(a, q)=1$, and $|\gamma q-a|<P^{-2-8}$. Then, for $i=1, \ldots, 10$,

and

$$
T_{i}(\gamma) \ll P|S(a, q)| q^{-1}\left(1+P^{3}\left|\gamma-a q^{-1}\right|\right)^{-1}+q^{2 / 3+s}
$$

$$
T_{i}(\gamma) \ll P q^{-1 / 3}\left(1+P^{3}\left|\gamma-a q^{-1}\right|\right)^{-1} .
$$

Proof. The first inequality follows easily from Lemma 7.11 of Hua [7]. The second then follows in a straightforward manner from Lemma 4.

Lemma 6. Suppose that $1 \leqslant i, j, k \leqslant 10$, and $r_{i}, r_{j}, r_{k}$ are distinct. Then

$$
\iint_{\mathfrak{m}}\left|T_{i}\left(\gamma_{i}\right)^{8} T_{j}\left(\gamma_{j}\right)^{8} T_{k}\left(\gamma_{k}\right)^{4}\right| d \alpha_{1} d \alpha_{2} \ll P^{13+4 \delta+8}
$$

Proof. Let

where

$$
\mathfrak{N}_{1}\left(b_{i}, b_{j}, q_{i}, q_{j}\right)=\left\{\left(\alpha_{1}, \alpha_{2}\right):\left|q_{r} \gamma_{r}-b_{r}\right|<P^{-2-\delta}(r=i, j)\right\},
$$

and

$$
q_{i}, q_{j} \leqslant P^{1-\delta}, \quad\left(b_{i}, q_{i}\right)=\left(b_{j}, q_{j}\right)=1,
$$

$$
\left|b_{r}\right| \leqslant 2\left(\left|c_{r}\right|+\left|d_{r}\right|\right) q_{r} \quad(r=i, j) .
$$

The $\mathfrak{M}_{1}\left(b_{i}, b_{j}, q_{i}, q_{j}\right)$ are clearly disjoint. Let $\mathfrak{M}_{1}$ denote the union of those $\mathfrak{M}_{1}\left(b_{i}, b_{j}, q_{i}, q_{j}\right)$ with $q_{i} q_{j}>P^{3 / 4}$, and let $\mathfrak{m}_{1}$ denote the set of those points of $\mathfrak{m}$ not in $\mathfrak{M}_{1}$. Note that $\mathfrak{m} \backslash \mathfrak{m}_{1}$ may be a proper subset of $\mathfrak{M}_{1}$. 
We first of all treat $m_{1}$. Let $\left(\alpha_{1}, \alpha_{2}\right) \in \mathfrak{m}_{1}$. By Dirichlet's theorem we may choose $b_{i}, b_{j}, q_{i}, q_{j}$ so that

$$
\left|q_{r} \gamma_{r}-b_{r}\right|<P^{-2-\delta}, \quad\left(q_{r}, b_{r}\right)=1, \quad q_{r} \leqslant P^{2+\delta} \quad(r=i, j) .
$$

Since $\left(\alpha_{1}, \alpha_{2}\right) \in(\eta, 1+\eta) \times(\eta, 1+\eta)$, where $\eta$ is given by $(3.8)$, it is easily deduced from (4.7) that (4.6) holds.

If

$$
q_{r}>P^{1-8} \quad(r=i \text { or } j),
$$

then, by Weyl's inequality (cf. Lemma 3.6 of Hua [7]),

so that

$$
T_{r}\left(\gamma_{r}\right) \ll P^{1+8}\left(P^{-1}+q_{r}^{-1}+q_{r} P^{-3}\right)^{1 / 4},
$$

If for $r=i$ or $j$

$$
T_{r}\left(\gamma_{r}\right) \ll P^{3 / 4+8} \text {. }
$$

$$
q_{r} \leqslant P^{1-\delta} \text { and } P^{1 / 4-\delta}<q_{r}^{1 / 3}\left(1+P^{3}\left|\gamma_{r}-b_{r} q_{r}^{-1}\right|\right),
$$

then by (4.3) we have (4.9) once more.

We will show that no other possibility can occur. Thus, by (4.9), for every $\left(\alpha_{1}, \alpha_{2}\right) \in \mathfrak{m}_{1}$,

\section{Hence}

$$
\min \left(\left|T_{i}\left(\gamma_{i}\right)\right|,\left|T_{j}\left(\gamma_{j}\right)\right|\right) \ll P^{3 / 4+8} .
$$

$$
\begin{aligned}
\iint_{\mathfrak{m}_{1}}\left|T_{i}\left(\gamma_{i}\right)^{8} T_{j}\left(\gamma_{j}\right)^{8} T_{k}\left(\gamma_{k}\right)^{4}\right| d \alpha_{1} d \alpha_{2} \\
\\
\ll P^{3+48} \int_{0}^{1} \int_{0}^{1}\left|T_{i}\left(\gamma_{i}\right) T_{j}\left(\gamma_{j}\right)\right|^{4}\left(\left|T_{i}\left(\gamma_{i}\right)\right|^{4}+\left|T_{j}\left(\gamma_{j}\right)\right|^{4}\right)\left|T_{k}\left(\gamma_{k}\right)\right|^{4} d \alpha_{1} d \alpha_{2} .
\end{aligned}
$$

Therefore, by Schwarz's inequality and Lemma 3,

$$
\iint_{\mathrm{m}_{1}}\left|T_{i}\left(\gamma_{i}\right)^{8} T_{j}\left(\gamma_{j}\right)^{8} T_{k}\left(\gamma_{k}\right)^{4}\right| d \alpha_{1} d \alpha_{2} \ll P^{13+4 \delta+8}
$$

We have to show that (4.8) or (4.10) are the only possibilities that can occur. Suppose they are not. Then

and

$$
q_{r} \leqslant P^{3 / 4-3 \delta} \quad(r=i, j)
$$

$$
\left|\gamma_{r}-b_{r} q_{r}^{-1}\right| \leqslant q_{r}^{-1 / 3} P^{-11 / 4-\delta} \quad(r=i, j) .
$$

Hence, by (3.6),

$$
\left.\begin{array}{l}
\alpha_{1}-\frac{b_{i} d_{j} q_{j}-b_{j} d_{i} q_{i}}{\left(c_{i} d_{j}-c_{j} d_{i}\right) q_{i} q_{j}} \\
\alpha_{2}-\frac{b_{j} c_{i} q_{i}-b_{i} c_{j} q_{j}}{\left(c_{i} d_{j}-c_{j} d_{i}\right) q_{i} q_{j}}
\end{array}\right\} \ll\left(q_{i} q_{j}\right)^{-1 / 3} P^{-5 / 2-2 \delta} .
$$

Thus, if $q_{i} q_{j} \leqslant P^{3 / 4}$, then by (3.10) and (3.11) $\left(\alpha_{1}, \alpha_{2}\right)$ is on a major arc, 
whilst if $q_{i} q_{j}>P^{3 / 4}$, then by (4.12) and (4.7) we have (4.5), that is, $\left(\alpha_{1}, \alpha_{2}\right) \in \mathfrak{M}_{1}$. In either case the assumption $\left(\alpha_{1}, \alpha_{2}\right) \in \mathfrak{m}_{1}$ is contradicted.

It remains to treat $\mathfrak{M}_{1}$. By Schwarz's inequality and Lemma 3,

$$
\int_{0}^{1} \int_{0}^{1}\left|T_{i}\left(\gamma_{i}\right)^{4} T_{j}\left(\gamma_{j}\right)^{4} T_{k}\left(\gamma_{k}\right)^{8}\right| d \alpha_{1} d \alpha_{2} \ll P^{10+8} \text {. }
$$

Hence, by Schwarz's inequality,

$$
\iint_{\mathfrak{M}_{1}}\left|T_{i}\left(\gamma_{i}\right)^{8} T_{j}\left(\gamma_{j}\right)^{8} T_{k}\left(\gamma_{k}\right)^{4}\right| d \alpha_{1} d \alpha_{2} \ll P^{5+8}\left(\iint_{\mathfrak{M}_{1}}\left|T_{i}\left(\gamma_{i}\right) T_{j}\left(\gamma_{j}\right)\right|^{12} d \alpha_{1} d \alpha_{2}\right)^{1 / 2} \text {. }
$$

Suppose that $b_{i}, b_{j}, q_{i}, q_{j}$ satisfy (4.5) and (4.6). Since $r_{i}$ and $r_{j}$ are distinct we can make a change of variables so that, by (4.4),

$\iint_{\mathfrak{M}_{1}\left(b_{1}, b_{j}, \alpha_{i}, q_{j}\right)}\left|T_{i}\left(\gamma_{i}\right) T_{j}\left(\gamma_{j}\right)\right|^{12} d \alpha_{1} d \alpha_{2} \ll \iint_{\mathfrak{M}_{1}\left(b_{i}, q_{i}\right) \times \mathbb{M}_{1}\left(b_{j}, q_{j}\right)}\left|T_{i}\left(\gamma_{i}\right) T_{j}\left(\gamma_{j}\right)\right|^{12} d \gamma_{i} d \gamma_{j}$,

where

$$
\mathfrak{M}_{1}(b, q)=\left\{\gamma:|q \gamma-b|<P^{-2-\delta}\right\} .
$$

By (4.2), (4.5), (4.15), and (4.16),

$$
\begin{aligned}
\iint_{\mathfrak{M}_{1}\left(b_{i}, b_{j}, q_{i}, q_{j}\right)}\left|T_{i}\left(\gamma_{i}\right) T_{j}\left(\gamma_{j}\right)\right|^{12} d \alpha_{1} d \alpha_{2} \\
\quad \ll \prod_{r=i, j} \int_{0}^{q_{r}^{-1} P^{-8-\delta}}\left(P^{12}\left|S\left(b_{r}, q_{r}\right)\right|^{12} q_{r}^{-12}\left(1+P^{3} \beta\right)^{-12}+q_{r}^{8+12 \varepsilon}\right) d \beta \\
\ll \prod_{r=i, j}\left(P^{9}\left|S\left(b_{r}, q_{r}\right)\right|^{12} q_{r}^{-12}+q_{r}^{7+128} P^{-2-\delta}\right) .
\end{aligned}
$$

Hence, by Lemma 4,

$$
\begin{aligned}
& \iint_{\mathfrak{M}_{1}\left(b_{b}, b_{j}, q_{j}, q_{j}\right)}\left|T_{i}\left(\gamma_{i}\right) T_{j}\left(\gamma_{j}\right)\right|^{12} d \alpha_{1} d \alpha_{2} \\
& \ll P^{18}\left|S\left(b_{i}, q_{i}\right) S\left(b_{j}, q_{j}\right)\right|^{12}\left(q_{i} q_{j}\right)^{-12}+P^{14}\left(q_{i}^{-4}+q_{j}^{-4}\right) .
\end{aligned}
$$

We recall that $\mathfrak{M}_{1}$ is the union of those $\mathfrak{M}_{1}\left(b_{i}, b_{j}, q_{i}, q_{j}\right)$ with $q_{i} q_{j}>P^{3 / 4}$. Hence, by (4.5), (4.6), (4.17), and the periodicity of $S(a, q)$ we have (the second term in (4.17), when summed over all $b_{i}, b_{j}, q_{i}, q_{j}$, is absorbed in the term $q=1$ )

$$
\iint_{\mathbb{M}_{1}}\left|T_{i}\left(\gamma_{i}\right) T_{j}\left(\gamma_{j}\right)\right|^{12} d \alpha_{1} d \alpha_{2} \ll P^{16}\left(\sum_{q<P} q^{-28 / 3} \sum_{\substack{a=1 \\(a, q) \sqcap 1}}^{q}|S(a, q)|^{12}\right)^{2} .
$$

By Lemmas 12 and 13 of Hardy and Littlewood [5],

and

$$
S\left(a, p^{t}\right)=p^{t-1} \quad(t=2,3, p \neq 3, p \nmid a)
$$

$$
|S(a, p)| \leqslant 2 p^{1 / 2} \quad(p \nmid a),
$$


and by (4.12) of Hardy and Littlewood [4] and (3.46) of Hardy and Littlewood [6],

Trivially

$$
S\left(a, p^{t}\right)=p^{2} S\left(a, p^{t-3}\right) \quad(t>3, p \nmid a) .
$$

$$
S\left(a, 3^{t}\right) \ll 3^{l-1} \quad(t=2,3) .
$$

Suppose that $\left(a_{1}, q_{1}\right)=\left(a_{2}, q_{2}\right)=\left(q_{1}, q_{2}\right)=1$. Then, by the Chinese remainder theorem,

Hence

$$
S\left(a_{1}, q_{2}\right) S\left(a_{2}, q_{2}\right)=S\left(a_{1} q_{2}+a_{2} q_{1}, q_{1} q_{2}\right) .
$$

is multiplicative. Thus

$$
\sum_{\substack{a=1 \\(a, q)=1}}^{q}|S(a, q)|^{12}
$$

$$
\sum_{q<P} q^{-28 / 3} \sum_{\substack{a=1 \\(a, q)=1}}^{q}|S(a, q)|^{12} \leqslant \prod_{p<P}\left(1+\sum_{1 \leqslant h<P} p^{-28 h / 3} \sum_{\substack{a=1 \\ p \nmid a}}^{p^{h}}\left|S\left(a, p^{h}\right)\right|^{12}\right) .
$$

If $h$ is of the form $3 l+1$ with $l \geqslant 0$, then by (4.20) and (4.21),

$$
p^{-28 h / 3} \sum_{\substack{a=1 \\ p \nmid a}}^{p^{\wedge}}\left|S\left(a, p^{h}\right)\right|^{12} \ll p^{-25 h / 3+24 l+6}=p^{-l-7 / 3} .
$$

If $h$ is of the form $3 l+m$ with $l \geqslant 0$ and $m=2$ or 3 , then by (4.19), (4.21), and (4.22),

$$
p^{-28 h / 3} \sum_{\substack{a=1 \\ p \nmid a}}^{p^{h}}\left|S\left(a, p^{h}\right)\right|^{12} \ll p^{-25 h / 3+24 l+12 m-12} \leqslant p^{-l-1} .
$$

Hence, by (4.23),

$$
\sum_{Q<P} q^{-28 / 3} \sum_{\substack{a=1 \\(a, q)=1}}^{q}|S(a, q)|^{12} \leqslant \prod_{p<P}\left(1+C p^{-1}\right)
$$

for a suitable positive constant $C$. Hence, by (4.18) and elementary prime number theory,

$$
\iint_{\mathfrak{M}_{1}}\left|T_{i}\left(\gamma_{i}\right) T_{j}\left(\gamma_{j}\right)\right|^{12} d \alpha_{1} d \alpha_{2} \ll P^{16+8} .
$$

Therefore, by (4.14),

$$
\iint_{\mathfrak{N}_{1}}\left|T_{i}\left(\gamma_{i}\right)^{8} T_{j}\left(\gamma_{j}\right)^{8} T_{k}\left(\gamma_{k}\right)^{4}\right| d \alpha_{1} d \alpha_{2} \ll P^{13+8} .
$$

This with (4.11) completes the proof of the lemma.

LemMa 7. On the hypothesis of Lemma 6,

$$
\iint_{\mathrm{m}}\left|T_{i}\left(\gamma_{i}\right)^{8} T_{j}\left(\gamma_{j}\right)^{6} T_{k}\left(\gamma_{k}\right)^{6}\right| d \alpha_{1} d \alpha_{2} \ll P^{18+4 \delta+8} .
$$


Proof. The proof is immediate from Lemma 6 and the trivial inequality

$$
\left|T_{j} T_{k}\right|^{2} \leqslant\left|T_{j}\right|^{4}+\left|T_{k}\right|^{4}
$$

LeMma 8. We have

$$
\iint_{\mathrm{m}}\left|T_{1}\left(\gamma_{1}\right) \ldots T_{10}\left(\gamma_{10}\right)\right|^{2} d \alpha_{1} d \alpha_{2} \ll P^{13+48+8}
$$

Proof. Since the ratios $r_{1}, \ldots, r_{10}$ arise from the set $\mathscr{A}$ of Lemma 1, no ratio is repeated more than four times and there are at least three different ratios. Thus, by several applications of the trivial inequality

$$
\left|z_{1} \ldots z_{m}\right| \leqslant\left|z_{1}\right|^{m}+\ldots+\left|z_{m}\right|^{m},
$$

we can always reduce to one of the two crucial cases, namely either Lemma 6 or Lemma 7. This easily gives the lemma.

Lemma 9. We have

$$
\iint_{\mathrm{m}}\left|T_{1}\left(\gamma_{1}\right) \ldots T_{12}\left(\gamma_{12}\right) U\left(\gamma_{13}\right) \ldots U\left(\gamma_{16}\right)\right| d \alpha_{1} d \alpha_{2} \ll P^{91 / 10+28+8} .
$$

Proof. The proof is immediate from Lemmas 2 and 8 and Schwarz's inequality.

\section{The major arcs}

The rest of the proof is only briefly sketched, as it follows in all essentials that of Davenport and Lewis.

Lemma 10. Suppose that $\left(\alpha_{1}, \alpha_{2}\right) \in \mathfrak{M}\left(a_{1}, a_{2}, q\right)$,

and

$$
\begin{gathered}
q_{i}=q_{i}\left(a_{1}, a_{2}, q\right)=q /\left(q, c_{i} a_{1}+d_{i} a_{2}\right), \\
\beta_{j}=\alpha_{j}-a_{j} q^{-1} \quad(j=1,2),
\end{gathered}
$$

Then

$$
\mu_{i}=c_{i} \beta_{1}+d_{i} \beta_{2} \text {. }
$$

$$
T_{i}\left(\gamma_{i}\right) \ll P q_{i}^{-1 / 3}\left(1+P^{3}\left|\mu_{i}\right|\right)^{-1} .
$$

Lemmas 10 and 11 are Lemmas 33 and 34 of Davenport and Lewis [3].

Lemma 11. On the hypothesis of Lemma 10,

$$
U\left(\gamma_{i}\right) \ll q_{i}^{-1 / 5} P^{4 / 5+8} \text {. }
$$

LeMma 12. We have

$$
\sum_{a_{1}, a_{2}}\left(q_{1} \ldots q_{12}\right)^{-1 / 3}\left(q_{13} \ldots q_{16}\right)^{-1 / 5} \ll q^{-9 / 5+8}
$$

and

$$
\sum_{a_{1}, a_{2}}\left(q_{1} \ldots q_{16}\right)^{-1 / 3} \ll q^{-7 / 3+8},
$$

where in each case the summation is over $1 \leqslant a_{1}, a_{2} \leqslant q$ with $\left(a_{1}, a_{2}, q\right)=1$. 
Proof. This follows closely that of Lemma 35 of Davenport and Lewis [3], the only difference being that their relation (119) is to be replaced by

$$
\theta_{1}+\ldots+\theta_{\nu}=24 / 5
$$

in the proof of (5.5) and by

in the proof of (5.6).

$$
\theta_{1}+\ldots+\theta_{\nu}=16 / 3
$$

Lemma 13. Let $\tau \geqslant 0$, let $\mu_{i}$ be given by (5.3), and let

Then

$$
\mathscr{D}(\tau)=\left\{\left(\beta_{1}, \beta_{2}\right): \max \left(\left|\beta_{1}\right|,\left|\beta_{2}\right|\right)>P^{\tau-3}\right\} .
$$

$$
\begin{gathered}
\iint_{\mathscr{D}(\tau)} \prod_{i=1}^{12}\left(P /\left(1+P^{3}\left|\mu_{i}\right|\right)\right) d \beta_{1} d \beta_{2} \ll P^{6-5 \tau}, \\
\int_{-\infty}^{\infty} \int_{-\infty}^{\infty} \prod_{i=1}^{12}\left(P /\left(1+P^{3}\left|\mu_{i}\right|\right)\right) d \beta_{1} d \beta_{2} \ll P^{6},
\end{gathered}
$$

and

$$
\int_{-\infty}^{\infty} \int_{-\infty}^{\infty} \Pi^{\prime}\left(P /\left(1+P^{3}\left|\mu_{i}\right|\right)\right) d \beta_{1} d \beta_{2} \ll P^{5},
$$

where $\Pi^{\prime}$ denotes a product over any eleven of $i=1, \ldots, 12$.

This can be shown in the same way as Lemma 36 of Davenport and Lewis [3].

LEMMA 14. The contribution of all the major arcs $\mathfrak{M}\left(a_{1}, a_{2}, q\right)$ with $q>P^{78}$ to the integral (3.9) is $\ll P^{46 / 5-8}$.

Proof. The proof follows immediately from (5.5), (5.9), and Lemmas 10 and 11.

Let

$$
\mathfrak{M}_{0}\left(a_{1}, a_{2}, q\right)=\left\{\left(\alpha_{1}, \alpha_{2}\right):\left|\alpha_{r}-a_{r} q^{-1}\right|<P^{\delta-3}(r=1,2)\right\}
$$

denote a contracted major arc.

LEMMA 15. The contribution of all the $\mathfrak{M}\left(a_{1}, a_{2}, q\right) \backslash \mathfrak{M}_{0}\left(a_{1}, a_{2}, q\right)$ with $1 \leqslant a_{1}, a_{2} \leqslant q \leqslant P^{7 \delta}$ and $\left(a_{1}, a_{2}, q\right)=1$ to $(3.9)$ is $\ll P^{46 / 5-5 \delta}$.

Proof. Let $\left(\alpha_{1}, \alpha_{2}\right) \in \mathfrak{M}\left(a_{1}, a_{2}, q\right) \backslash \mathfrak{M}_{0}\left(a_{1}, a_{2}, q\right)$. By Lemma 10 of Davenport [2], $U\left(\gamma_{i}\right) \ll q_{i}^{-1 / 3} P^{4 / 5}$. Thus, by (5.4) and (5.7) for a particular set $a_{1}, a_{2}, q$, the contribution is

$$
\ll P^{16 / 5}\left(q_{1} \ldots q_{16}\right)^{-1 / 3} \iint_{\mathscr{D}(\delta)} \prod_{i=1}^{12}\left(P /\left(1+P^{3}\left|\mu_{i}\right|\right)\right) d \beta_{1} d \beta_{2} .
$$

The lemma now follows from (5.6) and (5.8). 
LeMmA 16. The contribution of all the $\mathfrak{M}_{0}\left(a_{1}, a_{2}, q\right)$ with

$$
1 \leqslant a_{1}, a_{2} \leqslant q \leqslant P^{78}
$$

and $\left(a_{1}, a_{2}, q\right)=1$ to $(3.9)$ is

where

$$
P^{16 / 5} \mathrm{~S}\left(P^{78}\right) I(P)+O\left(P^{9}\right),
$$

$$
\begin{gathered}
\Im\left(P^{7 \delta}\right)=\sum_{q \leqslant P^{78}} \sum_{\substack{a_{1}=1 \\
\left(a_{1}, a_{2}, q\right)=1}}^{q} \sum_{\substack{a=1 \\
l}}^{q} \prod_{i=1}^{16}\left(q_{i}{ }^{-1} S\left(b_{i}, q_{i}\right)\right), \\
b_{i}=\left(c_{i} a_{1}+d_{i} a_{2}\right) /\left(q, c_{i} a_{1}+d_{i} a_{2}\right), \\
I(P)=\int_{\left|\beta_{1}\right|,\left|\beta_{2}\right|<P^{\delta-8}} I_{1}\left(\mu_{1}\right) \ldots I_{12}\left(\mu_{12}\right) d \beta_{1} d \beta_{2},
\end{gathered}
$$

and $I_{i}(\gamma)$ is the analogue of (3.3) with summation replaced by integration.

The proof of Lemma 16 follows, via (5.10), the argument of Lemmas 40 and 41 of Davenport and Lewis [3].

\section{The final stages of the proof}

Lemma 17. We have

$$
I(P)=C P^{6}+O\left(P^{6-58}\right),
$$

where $C$ is a positive constant.

Proof. The proof follows from (5.8) and the argument of Lemma 42 of Davenport and Lewis [3].

LeMma 18. Let

$$
\mathfrak{S}=\sum_{\substack{q=1 \\ q}}^{\infty} \sum_{\substack{a_{1}=1 \\\left(a_{1}, a_{2}, q\right)=1}}^{q} \sum_{a_{2}=1}^{q} \prod_{i=1}^{16}\left(q_{i}^{-1} S\left(b_{i}, q_{i}\right)\right)
$$

Then $\mathfrak{S}$ converges absolutely,

and

$$
\mathfrak{S}\left(P^{78}\right)=\mathfrak{S}+O\left(P^{-98}\right) \ll 1
$$

$$
\subseteq>0 \text {. }
$$

Proof. The absolute convergence and (6.2) follow from Lemma 4 and (5.6). It is easily seen that the $q$ th term of the series is a multiplicative function of $q$. Thus

$$
\mathfrak{S}=\prod_{p} \chi_{p}
$$

where

$$
\chi_{p}=1+\sum_{h=1}^{\infty} \sum_{\substack{a_{1}=1 \\ p \nmid\left(a_{1}, a_{2}\right)}}^{p^{h}} \sum_{\substack{a_{2}=1 \\ p^{n}}}^{-16 h} \prod_{i=1}^{16} S\left(c_{i} a_{1}+d_{i} a_{2}, p^{h}\right)
$$


By Lemma 4 and (5.6), $\left|\chi_{p}-1\right| \ll p^{-2}$. Thus for some $p_{0}$

$$
\prod_{p>p_{0}} \chi_{p}>\frac{1}{2} \text {. }
$$

For any given $p \leqslant p_{0}$, it follows by the usual arguments that $\chi_{p}>0$ provided that the equations (1.1) have a non-singular solution in the $p$-adic field. Since not more than six of the ratios $r_{1}, \ldots, r_{16}$ are the same, every form $\lambda F+\mu G$ with $\lambda, \mu \neq 0,0$ contains at least ten variables in the $p$-adic field. Thus the corollary to Theorem 1 of Davenport and Lewis [3] ensures that there is a non-singular solution in the $p$-adic field. Hence, by (6.4) and (6.5) we have (6.3).

By (3.9) and Lemmas 9, 14, 15, and 16,

$$
\mathscr{N}(P)=P^{16 / 5} \mathfrak{S}\left(P^{7 \delta}\right) I(P)+O\left(P^{46 / 5-\delta}\right) .
$$

By Lemmas 17 and 18 this is

$$
C \subseteq P^{46 / 5}+O\left(P^{46 / 5-\delta}\right)
$$

which tends to infinity as $P$ tends to infinity. This completes the proof of the theorem.

\section{REFERENCES}

1. R. J. Cook, 'Pairs of additive equations', Michigan Math. J. 19 (1972) 325-31.

2. H. Davenport, 'On Waring's problem for cubes', Acta Math. 71 (1939) 123-43.

3. - and D. J. LewIs, 'Cubic equations of additive type', Philos. Trans. Roy. Soc. London Ser. A 261 (1966) 97-136.

4. G. H. HARDY and J. E. LitTLEwOod, 'Some problems of "Partitio Numerorum": II. Proof that every large number is the sum of at most 21 biquadrates', Math. Z. 9 (1921) 14-27.

5. —_ 'Some problems of "Partitio Numerorum": IV. The singular series in Waring's problem', ibid. 12 (1922) 161-88.

6. __ 'Some problems of "Partitio Numerorum": VI. Further researches in Waring's problem', ibid. 23 (1925) 1-37.

7. L. K. HUA, Additive theory of prime numbers, Amer. Math. Soc. Transl. 13 (Providence, R.I., 1965).

\section{Department of Mathematics}

Imperial College

and

Queen's Gate, London SW7 2BZ

The University of Michigan Ann Arbor, Michigan 48104 